\title{
Solution structure of the N-terminal zinc binding domain of HIV-1 integrase
}

Mengli Cai, Ronglan Zheng, Michael Caffrey, Robert Craigie, G. Marius Clore and Angela M. Gronenborn, Nature Struct. Biol. 4, 567-577 (1997).

In this article which appeared in the July issue, an error resulted in lower resolution versions of the figures appearing in the journal. Figures adversely effected by this $(5,6$ and 8$)$ are printed below and on the next page.

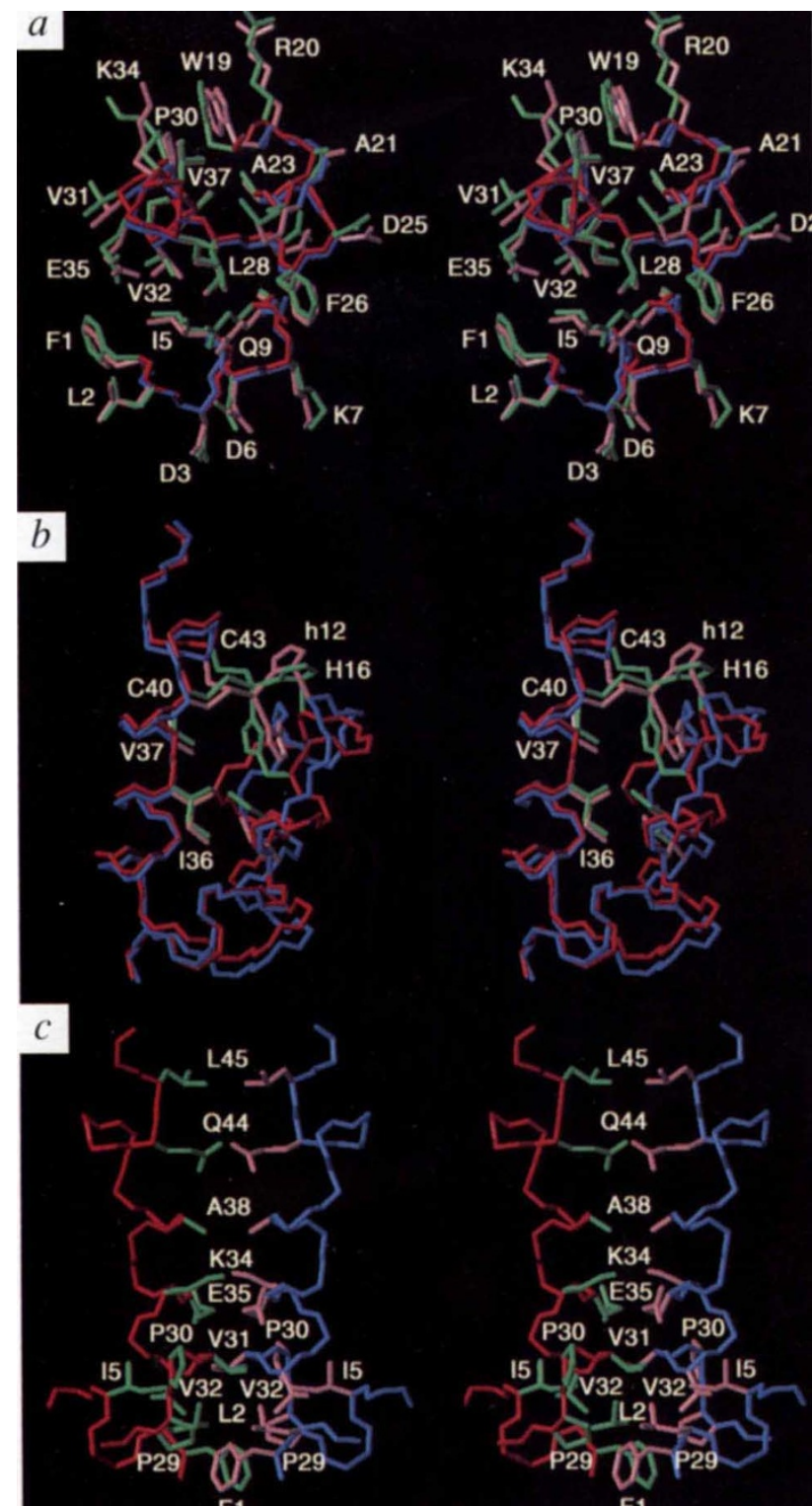

Fig. 8 Three views of a model of the integrase tetramer. The four subunits, A, B, C and $D$ are shown in yellow, red, green and blue respectively. The active site residues (Asp 64 and Asp 116) of each catalytic core are shown in magenta. (Note that the third catalytic residue, Glu 152 , is not visible in the electron density map). Views $(b)$ and $(c)$ are rotated by $90^{\circ}$ and $45^{\circ}$, respectively, relative to view (a); the rotation is about an axis that is parallel to the dimer interfaces of the two catalytic core dimers. The residues shown for the $\mathrm{N}$-terminal, catalytic and C-terminal domains are 1-47, 58-208 and 220-270 respectively. The coordinates for the X-ray structure of the catalytic core dimer and the NMR structure of the C-terminal domain dimer are taken from refs. 11 and 15 respectively (see figure on the right).
Fig. 5 Stereoviews showing superpositions of the restrained regularized mean structures of the $E$ and $D$ forms of $I^{1-55}$ for $\mathbf{a}$, the hydrophobic core and $\boldsymbol{b}$, the region surrounding the zinc. The backbone and sidechains are shown in red and green, respectively, for the E form, and in blue and pink, respectively for the $D$ form. In (b) $h 12$ refers to $H$ is 12 of the $D$ form, and $\mathrm{H} 16$ refers to His 16 of the $\mathrm{E}$ form. C. Stereoview of the dimer interface of $\mathrm{IN}^{1-55}$. The $\mathrm{E}$ form is displayed and the backbone of one subunit is shown in red and the other in blue; the corresponding sidechains are in green and pink respectively. The structure of the dimer interface is identical in the $E$ and $\mathrm{D}$ forms of $\mathrm{IN}^{\mathrm{T}-55}$ (see figure on the left).
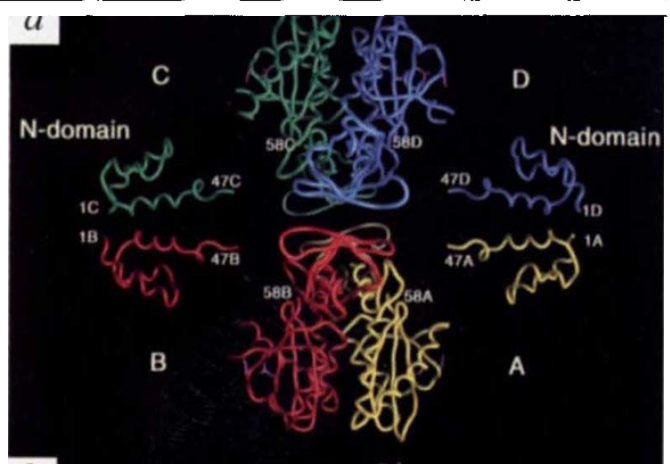

$b$

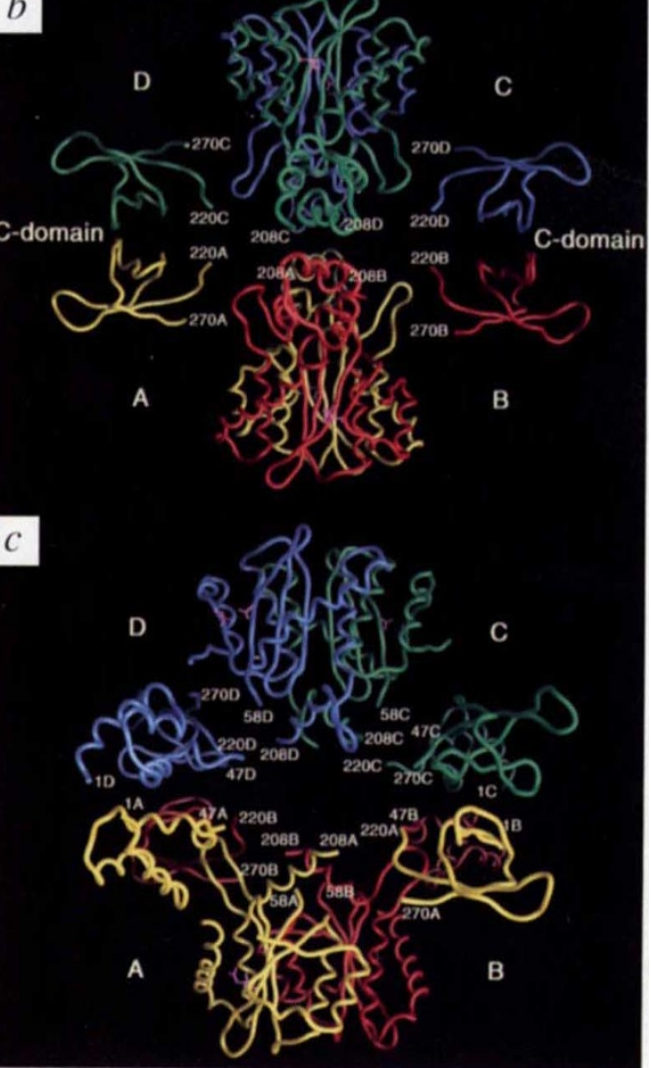




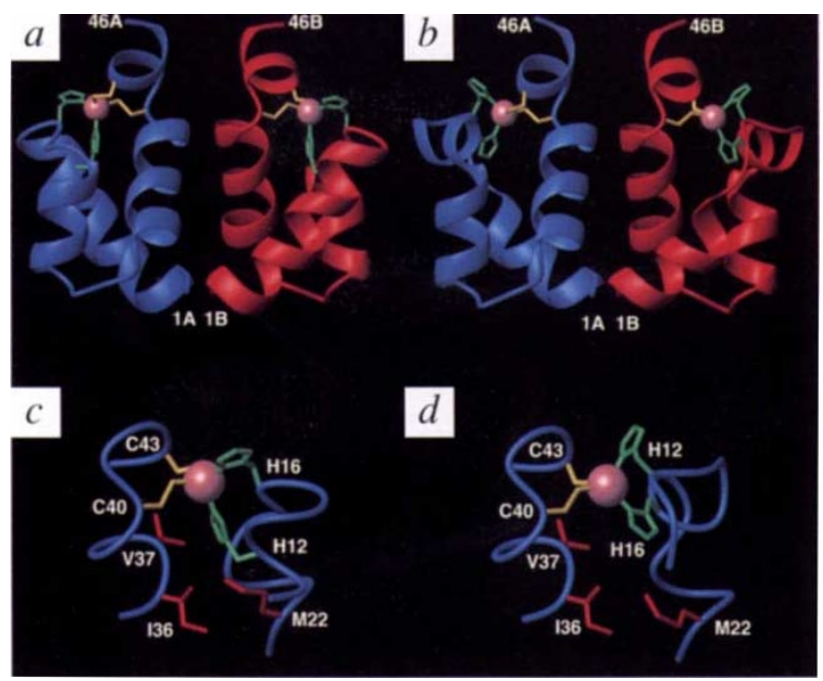

Fig. 6 Ribbon diagrams illustrating $\mathbf{a}$, the $E$ and $\boldsymbol{b}$, the $D$ forms of the $I^{1-55}$ dimer (residues 1-46). Detailed views of the region surrounding the zinc in $\boldsymbol{c}$, the $E$ and $\boldsymbol{d}$, the $D$ forms of $\mathrm{IN}^{1-55}$. The backbone of one subunit is shown in blue and of the other subunit in red, the zinc atom is displayed as a pink colored ball, selected sidechains are shown in red, and the coordinating cysteines and histidine residues are shown in yellow and green respectively.

\section{Bridging the gap}

Joel L. Sussman, Nature Struct. Biol. 4, 517 (1997).

On pg. 517 of the July issue, in the first sentence of the second paragraph, "Natural Center for Biotechnology Information" should read: National Center for Biotechnology Information.

\section{A low energy short hydrogen bond in very high resolution structures of protein receptor-phosphate complexes}

Zhongmin Wang, Hartmut Luecke, Nanhua Yao, and Florante A. Quiocho, Nature Struct. Biol. 4, 519-522 (1997).

On pg. 519 of the July issue, a (sign was inadvertently left out of the first line of the report, which should read: Sir - Short $(\leq 2.45 \AA)$ or low barrier hydrogen bonds (LBHBs), which possess strengths of as much as $30 \mathrm{kcal} \mathrm{mol}^{-1}$ for model compounds in the gas phase $^{1}$, have recently attracted considerable attention and controversy for their possible major role in enzyme catalysis ${ }^{1-5}$.

\section{corrigenda}

The following omissions were inadvertently made; below are the corrected items:

\section{The solution structure of the first $\mathrm{KH}$ domain of FMR1, the protein responsible for the fragile $X$ syndrome}

Giovanna Musco, Abdelhakim Kharrat, Gunter Stier, Franca Fraternali, Toby J. Gibson, Michael Nilges and Annalisa Pastore, Nature Struct. Biol. 4, 712-716 (1997).

On pg. 716 of the September issue, the Brookhaven Protein Data Bank code for the reported structure should read: $1 \mathrm{fmr}$.

\section{Three-dimensional structure of the Ras-interacting domain of RalGDS}

Lan Huang, Xiangwei Weng, Franz Hofer, G. Steven Martin and Sung-Hou Kim, Nature Struct. Biol. 4, 609-615 (1997).

The authors made an omission in their acknowledgements. They are also grateful to D. King of the Howard Hughes Medical Institute, University of California, Berkeley, for mass spectrometric work.

\section{The ion core in RNA folding}

Ignacio Tinoco, Jr. and Jeffrey S. Kieft, Nature Struct. Biol. 4, 509-512 (1997).

On pg. 510 in the July issue, Fig. $2 a$ is incorrect. The version to the right is as it should have been.

Fig. 2 a, Schematic of the P5b GAAA tetraloop docking in the $\mathrm{J} 6 \mathrm{a} / 6 \mathrm{~b}$ tetraloop receptor (light blue). An adenosine platform in the receptor allows continuous base stacking between the receptor and the tetraloop (dahsed box). Tertiary contacts, including an A-U-A base triple, further stabilize the interaction. A metal ion (red) is bound in the major groove of the P5b helix near two G०U base pairs. This figure is a slightly modified version of Fig. $4 b$ of ref. 4.

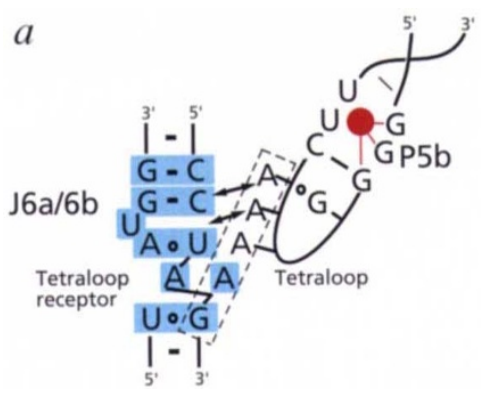

